\title{
Transmission Spectroscopy for the Assessment of the Yolk Index for White-Shelled Eggs and Brown-Shelled Eggs
}

\author{
Chia-Chun Lai ${ }^{1}$, Shan-Yu Jung ${ }^{1}$, Ching-Wei Cheng ${ }^{2 *}$ \\ ${ }^{1}$ Department of Bio-industrial Mechatronics Engineering, National Chung Hsing University \\ No.145 Xingda Rd., South Dist., Taichung City 402, Taiwan (R.O.C.) \\ s39911050@gmail.com \\ ${ }^{2}$ College of Intelligence, National Taichung University of Science and Technology, \\ No.129, Sec.3, Sanmin Rd, North Dist., Taichung City 404, Taiwan (R.O.C.) \\ cwcheng@nutc.edu.tw
}

\section{Extended Abstract}

Eggs are a source of protein, fat and micronutrients, they play an important role in basic nutrition [1], and the price is cheap. The freshness of eggs changes with processing, transportation and storage. The main factors that affect the freshness of eggs include: weight loss, increased air cells, egg yolk index, Huff units, protein $\mathrm{pH}$, and egg yolk $\mathrm{pH}$. In the detection of freshness, the methods of Haugh unit and egg yolk index are more commonly used for destructive testing. Nondestructive testing methods include electronic nose [2] and dielectric [3] measurement methods. In the egg yolk index, spectroscopy is rarely used as a detection method.

In this study, the full spectrum was used to detect the yolk index of white-shell eggs and brown-shell eggs. Use 100 eggs each for Roman white shell eggs and brown shell eggs. Eggs are stored in an environment with a temperature of 23 to $26^{\circ} \mathrm{C}$ and a humidity of 55\% RH for 9 days, and recorded once a day. The raw spectral response data of white and brown shell eggs vary between 500-810 $\mathrm{nm}$ [4], so 500-810 nm spectra will be used to analyze and record the height and diameter of the yolk. The egg measurement spectrum uses standard normal variation (SNV) to process the signal and multiple linear regression (MLR) and multilayer perceptron (MLP) are used to establish the correlation between the "spectrum and egg yolk index". And randomly take 30 eggs as prediction group for prediction.

The analysis results show that multiple linear regression uses five spectral wavelengths $(699 \mathrm{~nm}, 809 \mathrm{~nm}, 630 \mathrm{~nm}, 759$ $\mathrm{nm}$, and $794 \mathrm{~nm}$ ) to establish the correction equation for white-shelled eggs. The correlation coefficient (Rc) of the correction group was 0.857 . The correlation coefficient $(\mathrm{Rp})$ of the prediction group was 0.721 . Correction equation for MLP analysis of white shell eggs. The correlation coefficient $(\mathrm{Rc})$ of the correction group was 0.975 . The correlation coefficient (Rp) of the prediction group was 0.603. The multiple linear regression method was used to establish the correction equations for brown eggs with seven spectral wavelengths $(724 \mathrm{~nm}, 687 \mathrm{~nm}, 679 \mathrm{~nm}, 728 \mathrm{~nm}, 659 \mathrm{~nm}, 683 \mathrm{~nm}$ and $733 \mathrm{~nm}$ ). The correlation coefficient $(\mathrm{Rc})$ of the correction group was 0.815 . The correlation coefficient $(\mathrm{Rp})$ of the prediction group was 0.607 . The correction equation of brown shell eggs was established using MLP. The correlation coefficient $(\mathrm{Rc})$ of the correction group was 0.98 . The correlation coefficient $(\mathrm{Rp})$ of the prediction group is 0.64 , so the results show that the wavelength measured by the spectrum can effectively predict the egg yolk index.

\section{References}

[1] J.M. Miranda, X. Anton, C. Redondo-Valbuena, P. Roca-Saavedra, J.A. Rodriguez, A. Lamas, C. M. Franco, A. Cepeda. "Egg and Egg-Derived Foods: Effects on Human Health and Use as Functional Foods." Nutrients, vol. 7 , no.1, pp. 706-729, 2015.

[2] J. Li, S. Zhu, S. Jiang, \& J. Wang. "Prediction of egg storage time and yolk index based on electronic nose combined with chemometric methods." LWT-Food Sci. Technol., vol. 82, pp. 369-376, 2017.

[3] S. K. Lau, \& J. Subbiah. "An automatic system for measuring dielectric properties of foods: Albumen, yolk, and shell of fresh eggs." J. Food Eng., vol. 223, pp. 79-90, 2018.

[4] C. W. Cheng, S. Y. Jung, \& C. C. Lai. "Transmission spectral analysis models for the assessment of white-shell eggs and brown-shell eggs freshness.” J. Supercomput., vol. 76, pp. 1680-1694, 2020. https://doi.org/10.1007/s11227-01903008-Z 